\title{
Implicancias de las alteraciones en la función ventricular en pacientes con Accidente Cerebrovascular Isquémico.
}

\author{
Implications of alterations in ventricular function \\ in patients with Ischemic Stroke.
}

\author{
Gisela N. Saavedra ${ }^{1}$, Darío Tavolini ${ }^{1}$, Juan I. Jairala ${ }^{2}$. \\ ${ }^{1}$ Hospital Provincial del Centenario. Rosario; Argentina. \\ ${ }^{2}$ Facultad de Ciencias Médicas. Universidad Nacional de Rosario. Rosario; Argentina.
}

\begin{abstract}
Autor por correspondencia: Gisela N. Saavedra - gi_saavedr@hotmail.com Conflictos de intereses: no presenta.
\end{abstract}

\section{Resumen}

En este estudio se quiso demostrar el impacto de la Insuficiencia Cardiaca en pacientes que presentaron $\mathrm{ACV}$ isquémico, en la recuperación funcional y pronóstico durante la internación, tanto en pacientes con diagnóstico clínico de Insuficiencia Cardíaca como en pacientes asintomáticos, relacionando disfunción ventricular sistólica y diastólica.

Se realizó un estudio prospectivo, observacional. Se evaluaron 112 pacientes con ACV isquémico, se dividieron en tres grupos, según la función ventricular izquierda: pacientes con Función Ventricular Normal, pacientes con Función Sistólica Alterada y pacientes con Función Diastólica Alterada.

Se comparó en cada grupo la escala NIHSS (al ingreso, 48 hs y alta), el pronóstico funcional, complicaciones neurológicas y extra neurológicas.

El grupo con Función Sistólica Alterada se relacionó con mayor incidencia de complicaciones clínicas y neurológicas, también en el grupo con Función Diastólica Alterada, pero de menor proporción. En ambos grupos se observó mayor mortalidad, con respecto a pacientes con Función Ventricular Normal.

El grupo de pacientes con Función Sistólica Alterada presentó peor pronóstico funcional, con un empeoramiento durante la internación. Presentaron peor pronóstico de enfermedad inicial y de la recuperación evaluada a corto plazo, durante la internación.

El grupo de pacientes con Función Diastólica Alterada presentó un mal pronóstico funcional aunque de menor proporción que el anterior. Se asoció a un empeoramiento significativo durante la internación, tanto de los pacientes que presentaron buen pronóstico como malo.

Palabras claves: ACV. Insuficiencia Cardíaca. Pronóstico.

\begin{abstract}
This study wanted to demonstrate the impact of Heart Failure in patients who presented ischemic stroke, on functional recovery and prognosis during hospitalization, both in patients with a clinical diagnosis of Heart Failure and in asymptomatic patients, relating systolic and diastolic ventricular dysfunction.

A prospective, observational study was carried out. 112 patients with ischemic stroke were evaluated, they were divided into three groups, according to left ventricular function: patients with Normal Ventricular Function, patients with Altered Systolic Function and patients with Altered Diastolic Function.
\end{abstract}


The NIHSS scale (at admission, 48 hours and discharge), functional prognosis, neurological and extra neurological complications were compared in each group.

The group with Altered Systolic Function was associated with a higher incidence of clinical and neurological complications, also in the group with Altered Diastolic Function, but with a lower proportion. Higher mortality was observed in both groups, compared to patients with Normal Ventricular Function.

The group of patients with Altered Systolic Function presented a worse functional prognosis, with a worsening during hospitalization. They presented a worse prognosis of initial disease and of recovery evaluated in the short term, during hospitalization.

The group of patients with Altered Diastolic Function presented a poor functional prognosis, although in a lower proportion than the previous one. It was associated with a significant worsening during hospitalization, both in patients with a good and poor prognosis.

\section{Keywords: ACV. Heart Failure. Prognosis.}

\section{Introducción}

El Accidente Cerebrovascular (ACV) en Argentina, es la primera causa de discapacidad (1). Las muertes por eventos cerebrovasculares (sin diferenciar causas isquémicas de hemorrágicas), ocupa el cuarto lugar luego de los tumores, las enfermedades del sistema respiratorio y las enfermedades cardiológicas (2).

Varios trastornos cardíacos entre ellos, la Insuficiencia Cardiaca (IC), se han asociado con una severidad elevada, aumento de la mortalidad y resultados desfavorables en pacientes con ACV (3). Es una entidad con alta prevalencia, altos índices de hospitalización, invalidez y mortalidad.

El ACV isquémico es una complicación neurológica frecuente de la IC con comorbilidades que interactúan mutuamente $(4,5,6)$.

En pacientes con IC con Fracción de eyección reducida (IC-FEr) que sufren un ACV isquémico, tienen un riesgo 2 veces mayor de mortalidad que los pacientes con Fracción de eyección normal, aumenta el riesgo de discapacidad funcional moderada a grave después del ACV $(7,8,9)$.

En pacientes con Insuficiencia Cardiaca con Fracción de eyección preservada (IC-FEp) se demostró que presentan un peor pronóstico funcional en pacientes con ACV en el periodo de un año.

La disfunción diastólica ha emergido como una causa importante de IC—FEp, se demostró que es un predictor independiente de la mortalidad en pacientes que padecieron IAM o shock séptico, pero no se ha estudiado ampliamente su impacto sobre pacientes con ACV isquémico (10).

Basándose en estudios limitados retrospectivos y de cohortes, no hubo diferencias significativas entre ambos tipos de IC, se demostró que los pacientes con IC-FEr presentan una incidencia del $10 \%$ de ACV isquémicos y pacientes con IC-FEp un $8 \%$. Se demostró que cada uno se presenta como factor de riesgo independiente de peor resultado funcional a los 3 meses del ACV agudo.

Se han realizado pocos estudios correlacionando la repercusión de la disfunción ventricular tanto sistólica como diastólica en pacientes con ACV isquémico y estos fueron realizados a largo plazo. En los mismos se ha reportado una mayor severidad inicial en los pacientes con insuficiencia cardíaca y un peor pronóstico a 90 días aún en aquellos con función ventricular conservada (3).

En este estudio se propuso demostrar el impacto de la IC en pacientes que presentaron ACV isquémico, en la recuperación funcional y pronóstico durante la internación, tanto en pacientes con diagnóstico clínico de IC como en pacientes asintomáticos, relacionando disfunción ventricular sistólica y diastólica en el periodo agudo del ACV isquémico durante su internación.

Se plantearon como objetivos, determinar la prevalencia de las alteraciones de función ventricular en pacientes con ACV isquémico, ingresados en el Servicio de Neurología del Hospital del Centenario. Analizar la relación entre la alteración de la función ventricular y el pronóstico en pacientes con $\mathrm{ACV}$ isquémico. Como objetivo secundario, evaluar la relación entre la Fibrilación Auricular (FA) y las alteraciones halladas en el Ecocardiograma.

\section{Materiales y Métodos}

Se realizó un estudio prospectivo, observacional.

Se evaluaron pacientes con diagnóstico de ACV isquémico, ingresados por el Servicio de Neurología del Hospital Provincial del Centenario de la ciudad de Rosario y pacientes que durante la internación por otra causa clínica, presentaran ACV isquémico, en el periodo comprendido entre el 1 de Junio de 2014 hasta el 31 de Mayo de 2017. 
Se recabó de la historia clínica de cada paciente, datos filiatorios, factores de riesgos cardiovasculares, resultados de laboratorio, tomografía de cráneo sin contraste y neuroimagen de control, ecocardiograma, electrocardiograma, tratamiento farmacológico actual y estadía hospitalaria.

A todos los pacientes se realizó Escala NIHSS al ingreso, a las 48 hs y al alta hospitalaria.

Del total de pacientes, se dividieron en tres grupos, según la función ventricular izquierda:

- Pacientes con Función Ventricular Normal

- Pacientes con Función Sistólica Alterada

- Pacientes con Función Diastólica Alterada

Se definió IC Sistólica o IC con fracción de eyección reducida (IC-FEr) a quienes presenten síndrome clínico o asintomático con una FE < 40\%; IC Diastólica o IC con fracción de eyección preservada (IC-FEp) a quienes presenten síndrome clínico o asintomático, con una $\mathrm{FE} \geq 40 \% \mathrm{y}$ evidencia de disfunción diastólica $(11,12,13)$.

En cada grupo se evaluaron el pronóstico funcional teniendo en cuenta la Escala Rankin Modificada (ERM) y se tuvo en cuenta la variación de la escala durante el ingreso y el alta. Un puntaje de ERM de 0 a 2 representó una afección leve, y un puntaje de ERM de 3 a 6 un resultado desfavorable.

También se evaluó en cada grupo el tamaño de la lesión isquémica en TAC de Cráneo del ingreso, y se dividió en pacientes que presentaron una lesión mayor del 1/3 del territorio de la Arteria Cerebral Media, y pacientes con lesión menor del 1/3 del territorio de Arteria Cerebral Media. Esta medida se aplicó a todos los territorios arteriales.

Se tuvo en cuenta el tiempo de estadía hospitalaria y se subdividió en 3 grupos según días de internación: 0-4 días, 5-7 días y mayor de 8 días.

Se evaluó el grado de IC según su clasificación funcional: Estadio Asintomático: A - B y Estadio Sintomático: C-D (Anexo 1) (7).

Se consideraron en cada grupo las complicaciones neurológicas y extra neurológicas.

Como Variables Asociadas se consideraron: antecedentes de hipertensión arterial, diabetes, cardiopatía, arritmias, tabaquismo, alcoholismo, dislipemia, obesidad, ACV previo, enfermedad renal, respiratoria (EPOC), enfermedad neoplásica y valvulopatías (presentando los pacientes valvulopatía de grado leve) $(14,15,16,17,18)$.

Todos los pacientes firmaron consentimiento informado.

Se determinaron criterios de Inclusión: Diagnóstico de ACV isquémico, ingresado en el Servicio de Neurología del Hospital Provincial del Centenario y de pacientes que durante la internación por otra causa clínica, presentaran ACV isquémico. Pacientes iguales o mayores de 18 años de edad, capaces de proveer consentimiento informado para todos los procedimientos.

Los criterios de exclusión fueron: Pacientes menores de 18 años. Pacientes que no se realizaron ecocardiograma durante su internación.

Se utilizó en el análisis estadístico Microsoft Excel 2013, Versión 15.0. Se analizó la frecuencia de las distintas variables nominales y ordinales mediante porcentajes, en tanto que se expresaron las variables cuantitativas mediante media estadística.

\section{Resultados}

Se incluyeron 112 pacientes mayores de 18 años con diagnóstico de ACV Isquémico, de los cuales se observó un 58.9\% masculino, 41.1\% femenino.

Del total se dividieron en tres grupos según la afección de la función ventricular, un 58\% (65 pacientes) presentaron una Función Ventricular Normal, un 26\% (29 pacientes) presentaron una Función Diastólica Alterada (IC-FEp) y un 16\% (18 pacientes) con Función Sistólica Alterada(IC—FEr).

Se dividieron además en pacientes con lesión menor de 1/3 del territorio de ACM (91.1\%) y lesión mayor de 1/3 del territorio de ACM (8.9\%), con mayor proporción en el grupo de Función Sistólica Alterada del 17\% del total, 11\% en el grupo de Función Diastólica Alterada y un 7\% en Función Ventricular Normal.

Según el tiempo de estadía hospitalaria se diferencia en internación durante 0-4 días, que representa el 12,5\% (14 pacientes), internación durante 5-7 días, representa el 50\% (56 pacientes) e internación mayor de 8 días, que representa el 37,5\% (42 pacientes), con un tiempo medio de 10 días (8 a 50 días).

El rango etario de mayor frecuencia de ACV isquémico fue entre 50 a 69 años.

Dentro de los Factores de Riesgo Cardiovasculares, con mayor frecuencia se observó la 
hipertensión arterial 71.4\%, obesidad 51.8\%, tabaquismo 43.5\%, dislipemia 33.9\%, cardiopatía 20.5\% y ACV isquémico previo $16 \%$.

En cuanto a los pacientes con antecedentes de ACV isquémico, se determinó un total de 18 pacientes, de los cuales se estratifico según la proporción de pacientes en cada grupo, representando un 5\% en pacientes con Función Sistólica Alterada, 38\% de pacientes en el grupo de Función Diastólica Alterada, 55\% en Función Sistólica Normal, todos ellos con tratamiento antiagregante instaurado.

En cuanto a la clasificación etiológica de TOAST se describe en la Figura 1 su distribución.

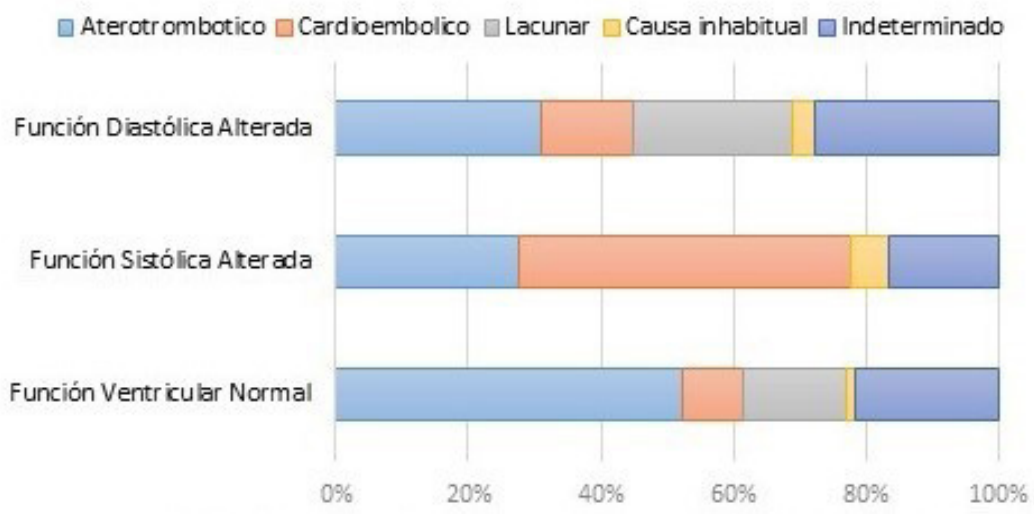

Figura 1: Distribución según Clasificación de TOAST y función ventricular.

Se estratificaron los pacientes según la clasificación funcional de IC, Estadio A: 37,5\%, Estadio B: 41.96\%, Estadio C: $17.86 \%$ y Estadio D: $2.68 \%$.

Dentro del grupo de cardiopatías, la más frecuente es el Infarto agudo de miocardio, representando un $32 \%$, la IC representa un $24.4 \%$ del total.

La FA representa el 26,66\% de las cardiopatías, y un 10,71\% de la población en total. Según los diferentes grupos, se diferenció en un 50\% en IC-FEr (la mitad de los casos presentaban antecedentes previos de FA, en tratamiento anticoagulante con rango terapéutico deficiente y el resto con diagnostico durante la internación), 33\% en IC-FEp (la mitad de los casos presentaban diagnóstico previo de FA, en tratamiento antiagregante, el resto fue detectado durante la internación) y 17\% en pacientes con Función Sistólica Normal (donde un paciente presentaba antecedentes previos de esta arritmia, en tratamiento antiagregante).

Las alteraciones ecocardiográficas relacionadas con esta etiología se observó: 41\% dilatación aurícula derecha, 33\% dilatación de cavidades izquierda e hipertrofia de VI y 16\% disfunción diastólica.

Del total de pacientes, un 34\% presentaron complicaciones sistémicas, siendo más frecuente la neumonía (13\%), síndrome febril (9\%), hiponatremia (5\%) y un 17\% complicaciones neurológicas, como transformación hemorrágica (9\%), edema maligno (6.5\%), convulsiones (2\%).

Las complicaciones tanto neurológicas como sistémicas se observaron en un 50\% en pacientes con IC-FEr, 37.9\% en pacientes con IC-FEp y un 27.7\% en pacientes con Función Ventricular Normal.

Se obtuvo de cada grupo una escala NIHSS promedio al ingreso, a las 48 hs y al alta, cuyos datos se encuentran representados en la Figura 2. 


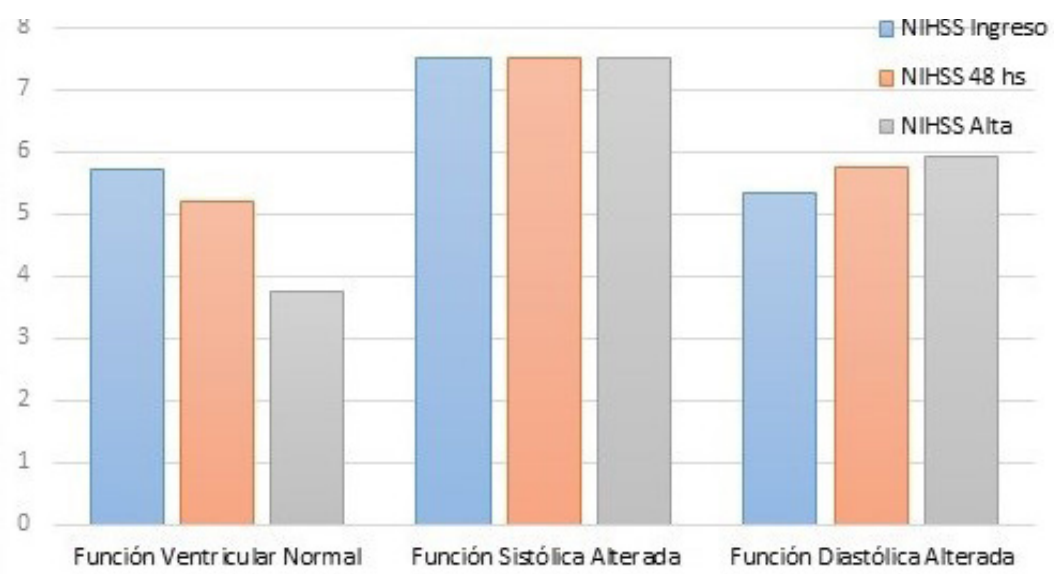

Figura 2: Distribución del promedio de Escala NIHSS al ingreso, 48 h y al alta según función ventricular.

Se diferenció en Escala de Rankin Modificada, en estadios con afección leve de 0 a 2 y en estadio con resultado desfavorable, de 3 a 6 , tanto al ingreso y al alta y su relación, representado en la Figura 3.

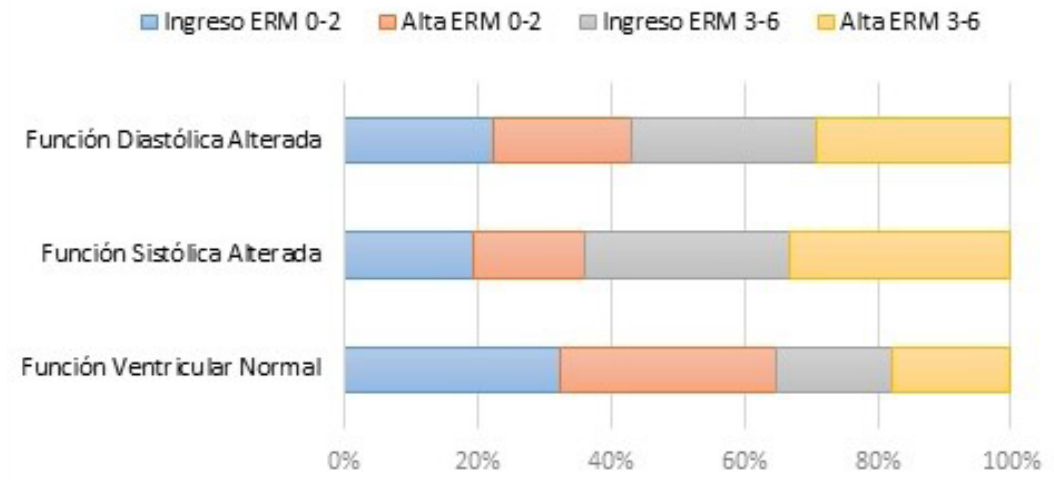

Figura 3: Distribución de ERM al ingreso y al alta según su función ventricular.

Del total de pacientes, un 8\% fallecieron (9 pacientes). Un paciente con Función Ventricular Normal, secundario a neumonía intrahospitalaria. Cuatro pacientes con IC-FEr, tres pacientes con lesión topográfica mayor, relacionado con edema maligno y neumonía broncoaspirativa, un paciente con lesión topográfica menor, complicado con shock cardiogénico. Cuatro pacientes con Función Diastólica Alterada, dos pacientes con lesión topográfica mayor, relacionado con edema maligno, y dos pacientes relacionados con síndrome febril sin causa justificada.

En cuanto a la relación entre el pronóstico, según ERM al ingreso y al alta con los días de estadía hospitalaria, se evidencia en las Figura 4 y 5.

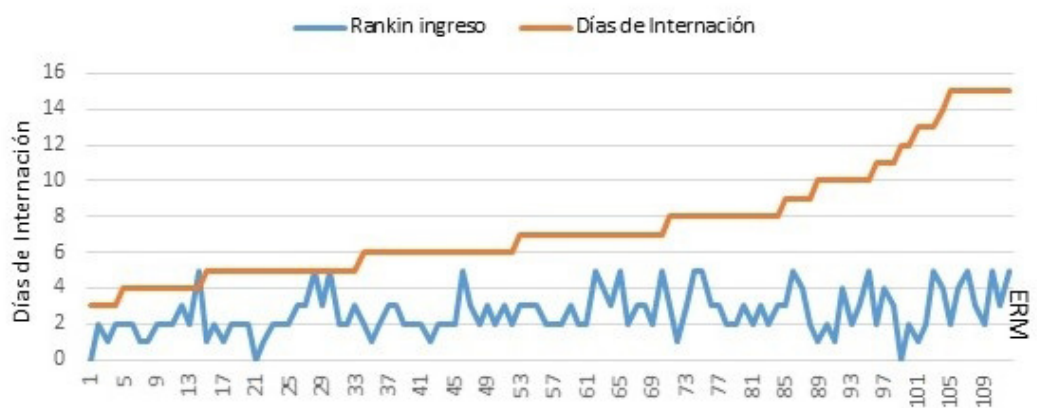

Figura 4: Relación entre días de internación y ERM al ingreso. 


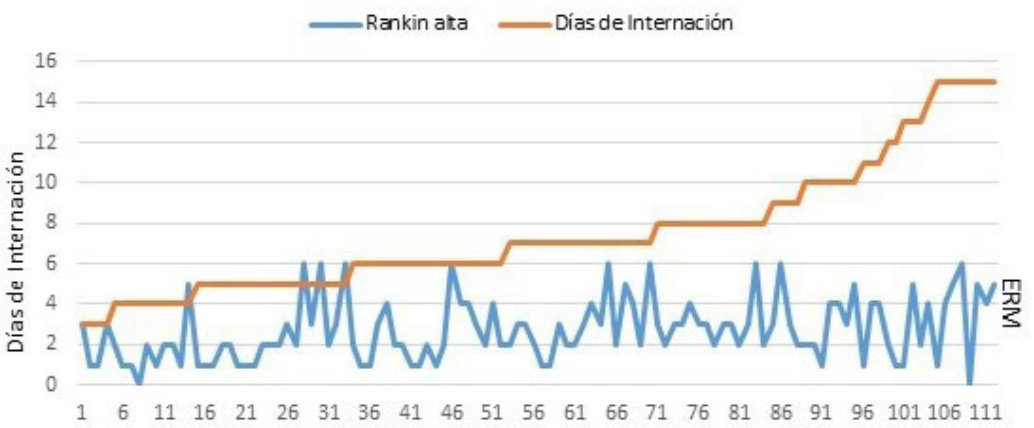

Figura 5: Relación entre días de internación y ERM al alta.

\section{Discusión}

La IC no solo aumenta el riesgo de incidencia de ACV isquémico, sino también la mortalidad y morbilidad de los pacientes $(3,4,7,10,19,20)$.

Estudios de cohortes retrospectivos han demostrado un riesgo de muerte después de un ACV más del doble para los pacientes con IC en comparación con los pacientes con ACV sin IC. Una posible explicación de este fenómeno podría ser un incremento de disfunción cardíaca inducida por accidente cerebrovascular debido a la desregulación autonómica. Los déficits neurológicos inducidos por ACV son más graves en los pacientes con IC. Además, los pacientes con ACV con IC tienen una estadía hospitalaria más prolongada que aquellos sin IC (4).

En este estudio se determinó la prevalencia de alteración ventricular en pacientes con ACV isquémico, la relación existente entre variables asociadas y su pronóstico funcional. Además la FA se ha establecido como un factor de riesgo independiente de ACV e IC, por lo que se determinaron variables asociadas a la misma.

Se observó que un 58\% de los pacientes presentaron una Función Ventricular Normal, y que en un $42 \%$ presentaron disfunción ventricular, en pacientes con ACV isquémico. De éstos, el 61\% presentaron Función Diastólica Alterada (IC-FEp) y el 39\% Función Sistólica Alterada (IC-FEr).

Seevidencióuna mayor proporción devaronesen un 58,9\% del total, novariandosignificativamente su proporción en los diferentes grupos.

La mayoría de los pacientes con alteración de la función diastólica (IC-FEp) se presentó en estadio asintomático según la clasificación funcional de IC, se evidenció una edad mayor, presentando un predominio etario entre 60 a 69 años.

Se diferencia de los pacientes con disfunción sistólica (IC-FEr) donde predomina el estadio C sintomático, con un predominio de edad más joven, entre 50 a 59 años. Esto se correlaciona con los datos demográficos nacionales en cuanto a la incidencia de ambos tipos de insuficiencia cardiaca (7).

En los pacientes con Función Ventricular Normal, se observó un predominio etario entre 60 a 69 años.

En cuanto al resultado de neuroimágenes, la mayoría de los pacientes presentó lesión tomográfica menor, en un $91.1 \%$.

El tiempo de internación entre 5 a 7 días predominó en los tres grupos evaluados.

En el grupo de pacientes con IC-FEr se observó una gran incidencia de patologías clínicas o neurológicas intercurrentes, representando un 50\%. En el grupo con IC-FEp un 37,9\% y en el grupo con Función Ventricular Normal un 27,7\%.

Se demostró que el 34\% del total presentó complicaciones sistémicas, siendo la más frecuente la infección pulmonar (neumonía broncoaspirativa y neumonía intrahospitalaria), con un leve predominio en pacientes con Función Ventricular Normal e IC-FEp. Esto se asoció a aumento de estadía hospitalaria.

Las complicaciones neurológicas se presentaron en un 17\%, se relaciona con un impacto negativo a corto plazo en el resultado funcional y en la mortalidad, se observó en lesiones isquémicas mayores al 1/3 del territorio de la ACM, se evidenció edema cerebral, transformación hemorrágica, sin gran diferenciación entre grupos, relacionado a etiología cardioembólica y aterotrombótica de grandes $\operatorname{vasos}(21)$.

Las etiologías que más se relacionan a lesiones isquémicas mayores son la Cardioembolia (relacionado a pacientes con IC o FA) y Aterotrombótica de grandes vasos (embolia arteria—arteria), 
esto además de provocar disfunción neurológica importante por el área comprometida, durante su evolución en las primeras 72 hs son más propensos a desarrollar un aumento del edema cerebral, provocando mayor deterioro neurológico $(22,23,24)$. Además cuanto mayor es el área del infarto, mayor probabilidad de que se produzca transformación hemorrágica y con esto su empeoramiento clínico $(25,26,27)$.

En nuestra muestra, la mortalidad intrahospitalaria fue de un $8,03 \%$. Se describieron un total de 9 fallecidos, de los cuales el 88\% (8 pacientes) están relacionados con disfunción ventricular de igual proporción tanto en IC-FEr como en IC-FEp, relacionándose además con lesión isquémica mayor (28). Las causas de mortalidad del paciente con Función Ventricular Normal fue una complicación infecciosa, neumonía intrahospitalaria, asociado a internación prolongada.

Las enfermedades cardíacas representan un 34,82\% del total de pacientes, siendo la cardiopatía isquémica la más frecuente (este hallazgo podría ser un sesgo por ser el Hospital en estudio, un centro de derivación para la patología especifica), seguido de FA y la IC sintomática, esta última en un 24,2\%, con un predominio del grupo IC-FEr $(28,29)$.

La FA representó un 10,71\% del total. Se asoció el 50\% con IC-FEr el 33\% con IC—FEp y el 17\% en pacientes con Función Ventricular Normal. Aproximadamente la mitad de los casos presentaron antecedentes previos de FA, donde un poco porcentaje (un tercio) estaba en tratamiento anticoagulante previo a la internación, siendo éste el tratamiento más eficaz contra la recurrencia de ACV isquémico en esta patología.

La IC es uno de los predictores más fuertes de FA y aumenta su riesgo hasta seis veces, está presente en el 15\% al 30\%. La prevalencia de FA aumenta con la clase funcional, o sea que a mayor gravedad de la IC mayor es la incidencia de esta arritmia $(30,31)$.

La FA es frecuente en los pacientes con IC—FEr y más aún en los pacientes con IC—FEp.

Se describe en estudios que aproximadamente el 15\% de todos los ACV presentan FA y la coexistencia entre ACV, FA e IC se produce en un $2 \%$ (32).

Se determinó que en pacientes con IC y ritmo sinusal, se encontraron dos variables que pueden predecir la aparición de FA, en primer lugar, la presencia de FA paroxistica y, en segundo lugar, la reducción de la contribución de la aurícula izquierda al llenado ventricular. El problema de estas variables fue su baja especificidad, menor del $20 \%$.

En nuestra población, las alteraciones ecocardiográficas relacionadas más frecuentemente fueron la dilatación auricular derecha, seguido de hipertrofia de VI, dilatación de cavidades izquierdas y alteración en la función diastólica.

Es de importancia destacar dentro del grupo de IC-FEp una mayor proporción de ACV indeterminado (del subtipo de origen desconocido) con respecto a otros grupos. Es importante mencionar que en el grupo con Función Sistólica Normal, predominó el tipo de ACV indeterminado por presentar estudios incompletos.

Variosestudiosdemostraronqueun 5a20\%delospacientescon ACVindeterminado(Criptogenéticos) pueden tener FA paroxística en la monitorización a largo plazo, a partir de estos hallazgos nos obliga sospechar en FA como causa mayor en este subgrupo (33).

Además la FA es la causa más común de ACV cardioembólico y está estrechamente relacionada con la disfunción diastólica, lo que sugiere un aumento del riesgo de ACV recurrente en esta población, por lo que es importante sospechar esta entidad así determinar el tratamiento preventivo secundario adecuado $(34,35,36)$.

Si bien se observó mayor incidencia de ACV recurrente en el grupo de Función Ventricular Normal, el grupo de Alteración de la Función Diastólica presento un porcentaje no despreciable, un 38\%, en comparación al grupo de Alteración de la Función Sistólica (5\%), ningún paciente se relacionó con diagnostico o antecedentes de FA. En nuestro estudio, es difícil extrapolar con la pequeña muestra, pero dada las características, es importante remarcar dicha tendencia.

En cuanto al pronóstico, se observó que en el grupo de pacientes con función sistólica conservada, la situación funcional tanto del ingreso como en el alta fue buena, con predominio de puntajes bajos en la ERM (1 y 2) en el 65\%, presentando mayor proporción de pacientes con mejoría de estado funcional. Esto también se ve reflejado en la variación de la escala NIHSS donde se evidenció puntajes por debajo de 6, con una tendencia a recuperación comparando los puntajes a las 48 hs y al alta.

A diferencia de esto, los pacientes con IC-FEr, presentaron peor pronóstico funcional, con predominio en puntajes altos de ERM (3 al 6) del 62\%, con un empeoramiento durante la internación donde se comparó la funcionalidad al ingreso y al alta. Por lo que se demostró que no sólo presentan peor pronóstico de enfermedad inicial, sino que presentan peor pronóstico de recuperación evaluada a 
corto plazo, durante la internación. En la variación de la escala NIHSS se evidencia un puntaje promedio mayor, presentando poca variación proporcional durante estadía y alta.

Es importante remarcar la tendencia del grupo de IC-FEp, donde presentó un peor pronóstico funcional, aunque de menor proporción que el grupo anterior (59\%). Se evidencio puntaje alto en ERM (3 a 6) con un franco empeoramiento durante la internación, tanto de los pacientes que presentaron buen pronóstico como malo. Esto se demostró en la variación de la escala de NIHSS, que demuestra la tendencia hacia el empeoramiento funcional, de menor magnitud que en el grupo de Función Sistólica Alterada.

En relación a la estadía hospitalaria, sólo se demostró un mejor pronóstico en estadía corta (menor a 4 días), sin grandes diferencias en cuanto a pronóstico y funcionalidad entre grupos de internación media y prolongada.

Cabe destacar que el único tratamiento farmacológico que reduce la discapacidad en el ACV isquémico agudo es el uso de terapia trombolítica endovenosa, no disponible en nuestro medio (36).

Corroboramos la asociación con peor pronóstico en pacientes que presentan tanto IC-FEr como IC-FEp, incluyendo a pacientes en estadios asintomáticos, donde se demostró mayor incidencia de complicaciones clínicas y neurológicas, mayor mortalidad y peor resultado funcional inicial, con una tendencia a empeoramiento durante la internación. Esto se debería tener en cuenta en un estudio a futuro a fin de reevaluar los posibles tratamientos y cuidados de estos pacientes.

\section{Agradecimientos}

A nuestros compañeros, colegas y maestros.

Fuente de financiamiento: no presenta.

\section{Referencias bibliográficas}

1. Pigretti S, Alet M, Mamani C, et al. Consenso sobre Accidente Cerebrovascular Isquémico agudo. Medicina (Buenos Aires) 2019 ; Vol. 79 (Supl. II): $1-46$.

2. Eswardson M, Dromerick A, Kasner S, Dashe J. Ischemic stroke prognosis in adults.Updated: Jan 06, 2017.

3. Ois A, Gomis M, Cuadrado Godia M, et al. Heart failure in Acute ischemic Stroke. J Neurol 2008 255:385-389.

4. Haeusler K, Laufs U, Endres M. Chronic Heart Failure and Ischemic Stroke. Stroke 2011; 42:2977-2982.

5. Witt B, Gami A, Ballman K, et al. The Incidence of Ischemic Stroke in Chronic Heart Failure: A Meta—Analysis. Journal of Cardiac Failure Vol. 13 No. 6. 2007.

6. Scherbakov N, Haeusler K, Doehner W. Ischemic stroke and heart failure: facts and numbers. Heart Failure 2015; 2: 1-4.

7. Consenso de Insuficiencia Cardiaca Crónica. Sociedad Argentina de Cardiología. Vol. 84 Suplemento 3 Octubre, 2016.

8. Burkot J, Kopec G, Pera J, Slowik A, Dziedzoc T. Decompensated Heart Failure Is a Strong Independent Predictor of Functional Outcome After Ischemic Stroke. Journal of Cardiac Failure Vol. 21 No. 8, 2015.

9. Ganiger I. Assessment of Left Ventricular Function by ECG \& 2DECHO in Acute Ischemic Stroke. Journal of Science. Vol 5. Issue 6. 2015. 409-41.

10. Ryu W, Park J, Ko S, et al. Diastolic Dysfunction and Outcome in Acute Ischemic Stroke.Cerebrovascular Disease 2016; 41(3-4):148—55.

11. Marino J, Barisani J, Thierer J, et al. Consenso de insuficiencia cardiaca crónica. Revista argentina de cardiología / VOL 84 suplemento 3 / octubre 2016 .

12. Barisani J, Fernandez A, Thierer J, et al. Consenso de Insuficiencia Cardíaca con Función Sistólica Preservada. Revista argentina de cardiología / VOL $78 \mathrm{~N}^{\circ} 4$ / julio-agosto 2010.

13. Tacchi H. Disfunción sistólica ventricular izquierda asintomática. Instituto Alexander Fleming. Ciudad de Buenos Aires. República Argentina.

14. Jauch E, Saver J, Adams H, et al. Guidelines for the Early Management of Patients With Acute Ischemic Stroke A Guideline for Healthcare Professionals From the American Heart Association/American Stroke Association. 2013.

15. Méndez J, Leiguarda R, Ameriso S. Enfermedades cerebrovasculares isquémicas y hemorrágicas. Segunda edición.

16. Biller J, Love B, Schneck M. Enfermedades vasculares del sistema nervioso. Enfermedad vascular cerebral isquémica. Neurología clínica. Volumen 2. Quinta edición.

17. O'Donnell M, Xavier D, Liu L, et al. Risk factors for ischaemic and intracerebral haemorrhagic stroke in 22 countries: a case—control study. Lancet 2010: 376; 112-23.

18. Arboix A. Cardiovascular risk factors for acute stroke: Risk profiles in the different subtypes of ischemic stroke World. J Clin Cases 2015 May $16 ; 3(5): 418-429$.

19. Kang S, Kim J, Park J,et al. Kang S, Kim J, Park J,et al. Risk of Stroke in Congestive Heart Failure With and Without Atrial Fibrillation. International Journal of Cardiology. Volumen 248, P182-187,01 de diciembre de 2017.

20. Katsanos A, Parissis J, Frogoudaki A, et al. Heart failure and the risk of ischemic stroke recurrence: A systematic review and meta—analysis. J 
Neurol Sci. 2016 Mar 15; 362:182

21. Barrett K, Khatri P, Jovin T. Complications of Ischemic Stroke: Prevention and Management. Continuum Lifelong Learning Neurol 2008;14(6):61-79.

22. Amarenco P, Bogousslavsky J, Caplan L, Donnan, Hennerici M. Classification of Stroke Subtypes. Cerebrovasc Dis 2009;27:493-501.

23. Díez-Tejedor E, Del Brutto O, Álvarez-Sabín J, Muñoz M, Abiusi G. Classification of the Cerebrovascular Diseases. Iberoamerican Cerebrovascular Diseases Society. Rev Neurol 2001; 33: 455-64.

24. Rodríguez-Yáñez M, Fernández Maiztegui C, Pérez—Concha T, et al. Enfermedades vasculares cerebrales Neurologia. Quinta edición. J. J. Zarranz.

25. Weimar C, Mieck T, Buchthal J, et al. Neurologic worsening during the acute phase of ischemic stroke. Arch Neurol 2005; 62(3):393-397.

26. Heiss W, Kidwell C, Imaging for Prediction of Functional Outcome and Assessment of Recovery in Ischemic Stroke. Stroke. 2014;45:00-00.

27. Pexman J, Barber P, Hill M, et al. Use of the Alberta Stroke Program Early CT Score (ASPECTS) for Assessing CT Scans in Patients with Acute Stroke. AJNR Am J Neuroradiol 22:1534-1542, September 2001

28. Garcia M. Diagnóstico y guía terapéutica de la insuficiencia cardíaca diastólica. Rev Esp Cardiol 2003;56(4):396 - 406

29. Barisani J, Fernandez A, Thierer J, et al. Consenso de Insuficiencia Cardíaca con Función Sistólica Preservada. Revista argentina de cardiología / VOL $78 \mathrm{~N}^{\circ} 4$ / julio-agosto 2010

30. Tacchi H. Disfunción sistólica ventricular izquierda asintomática. Instituto Alexander Fleming. Ciudad de Buenos Aires. República Argentina.

31. Abdul-Rahim A, Perez A, MacIsaac R, et al. Risk of stroke in chronic heart failure patients with preserved ejection fraction, but without atrial fibrillation: analysis of the CHARM—Preserved and I—Preserve trials. Eur Heart J. 2017 Mar 7;38(10):742—75.

32. Castellanos R, Lobo Márquez L, González S. Fibrilación auricular en pacientes con insuficiencia cardíaca. Rev Fed Arg Cardiol 2003; 32: $351-357$.

33. Seo J, Lee K, Lee J, et al. Implication of left ventricular diastolic disfunction in criptogenic isquemic stroke. Stroke. 2014; 45:2757-2761.

34. Tiwari S, Schirmer H, Jacobsen B, et al. Association between diastolic dysfunction and future atrial fibrillation in the Tromsø Study from 1994 to 2010 Heart 2015;101:1302-1308.

35. Saposnik G, Hill M, O’Donnell M, Fang J, Hachinski V, Kapral M. Registry of the Canadian Stroke Network for the Stroke Outcome Research Canada (SORCan) Working Group. Variables associated with 7—day, 30—day and 1-year fatality after ischemic stroke. Stroke 2008; 39: 2318— 24.

36. Powers W, Derdeyn C, Biller J, et al. AHA/ASA Guideline 2015 AHA/ASA Focused Update of the 2013 Guidelines for the Early Management of Patients With Acute Ischemic Stroke Regarding Endovascular Treatment. A Guideline for Healthcare Professionals From the American Heart Association/American Stroke Association. Stroke. 2015; 46:000-000.

\section{Anexo 1: Insuficiencia Cardiaca según su clasificación funcional (ACC/AHA):}

Estadio A: Asintomática. Sin daño estructural o alteración funcional del corazón. En riesgo de desarrollar IC. Factores de riesgo para el desarrollo de cardiopatía estructural.

Estadio B: Asintomática. Alteración estructural fuertemente relacionada con el desarrollo de IC. Disfunción ventricular asintomática, remodelamiento ventricular, hipertrofia ventricular, infarto previo, enfermedad valvular asintomática, cardiopatía congénita y cardiopatía chagásica.

Estadio C: IC sintomática. Alteración estructural cardíaca asociada a síntomas. Disnea, fatiga, tolerancia al ejercicio reducida, síntomas y signos congestivos.

Estadio D: IC Avanzada. Alteración estructural cardíaca severa. Sintomatología en reposo a pesar de máximo tratamiento. IC refractaria, internaciones frecuentes, puede requerir intervenciones especiales (inotrópicos, asistencia circulatoria y/o trasplante). 